This step requires external power to pump out the ions as they scatter and become trapped in the barrier. As a pump, the first experiments will use charge exchange of trapped ions with a neutral-atom beam which is injected into the barrier region at a pitch angle corresponding to no trapping in the local mirror field.

With the barrier in place and local heating applied, $T_{\text {ep }}$ can increase. As illustrated in Fig. 3, an increasing ion density $n_{\mathrm{p}}>n_{\mathrm{b}}$ will generate a local potential $\phi_{b}+\phi_{i}$ that scales with $T_{\text {ep }}$. In effect, the heating power applied to the plugs tends to push electrons out of that region, and the well depth $\phi_{\mathrm{b}}+\phi_{\mathrm{i}}$ increases accordingly to preserve charge neutrality.

The first real test of thermal barrier effectiveness will come in an upgrade of TMX in early 1982. Current theoretical studies include calculations of electron-power transfer rates, ion filling rates and charge exchange pumping effectiveness, electron cyclotron heating methods to raise $T_{\text {an }}$ and of course, the stability properties of this new configuration.

In most other respects, the TBTM resembles the conventional TM and the considerations of the preceeding section hold. As an indication of TBTM reactor possibilities, Fig. 4 shows the calculated reactor $Q$ versus the attainable $\beta$ for the conditions listed. Based on these results, an economic $Q \cong 10$ would require either $\beta \cong 0.5$ at the lower magnetic field, or at lest $\beta_{\mathrm{c}} \cong 0.3$ at the higher.

\section{Conclusions}

The expected improvements from singlecell mirror to tandem mirrors to tandems with thermal barriers are large, and the source of renewed interest in this fusion development line is clear. Experimentally, the TM programe is in its relative infancy. However, the concept is rooted in extensive mirror experience, and the first generation of tandem devices demonstrated their superiority. New ideas are being analyzed, e.g., improved pumping techniques and alternative magnetic configurations that are directed toward increased axisymmetry. The next few years should bring very rapid progress.

\section{FURTHER READING}

For an excellent 20 year history of the mirror programme, see Ryutov D.D., Nuclear Fusion 20 (1980) 1068.

For reports on progress in the major experiments, see the proceedings of the Biennial IAEA Conferences on Plasma Physics and Controlled Nuclear Fusion Research.

For a description of thermal barriers, see Baldwin D.E. and Logan B.G., Phys. Rev. Lett. 43 (1979) 1318.

For details of conceptual reactor designs, see the LLNL Reports: "Tandem Mirror Reactor with Thermal Barriers", UCRL-84255 (1980) by Carlson G.A. et al., and "Status Report on the Fusion Breeder", UCRL-84436 (1980) by Moir R.W.

\title{
Primordial Helium
}

\section{H. Andrillat, Montpellier}

(Astronomy Laboratory, University of Languedoc)

One of the most dependable guides for interpreting cosmological data continues to be the general theory of relativity based upon a representation of the real by a four dimensional continuum, the space-time, with a curvature that is variable with time. These days, Friedmann's models are generally accepted, where the function $R(t)$ expressing the curvature is one of the three forms shown in Fig. 1.

Despite the uncertainties which still exist on the geometric nature of the Universe, through a lack of sufficiently precise or extensive measurements, there is a convergence of all these models on a singularity with $R=0$ and a primordial explosion $(d R / d t=\infty)-$ the big bang. This resulted in the Universe having an initial temperature of extreme magnitude, since the curvature is related to the radiation temperature $T(t)$ by the expression:

$R T=$ cte $; T \rightarrow \infty$ when $R \rightarrow 0$.

\section{Primordial Energy and Particles}

All elementary particles and, in particular, the heavy particles, the hadrons could be created out of the thermal energy of the primordial Universe. For a particle of mass $m$, the threshold for such creation is given by the equation: $m c^{2}=k T$ where $k$ is Boltzmann's constant

$$
\left(=1.38 \times 10^{-23} \mathrm{~J} / \mathrm{K}\right)
$$

and $c$ the speed of light $\left(=3 \times 10^{8} \mathrm{~m} / \mathrm{s}\right)$.

The threshold temperature for the creation of a proton $\left(m_{\mathrm{p}}=1.67 \times 10^{-27} \mathrm{~kg}\right)$ is thus $10^{13} \mathrm{~K}$.

When the temperature of the Universe was very much greater than the threshold temperature for the production of a given particle $P$, particles of this type would be formed in very large numbers, with an equal number of antiparticles $\mathrm{P}^{\prime}$. Moreover, their mean free path would be very short as the density of matter, in this closely confined Universe so soon after its birth, would be huge. Consequently, particles and antiparticles annihilated each other as soon as they were formed, in equilibrium with the $\gamma$-radiation that created them and in a state of perfect exchange between matter and energy.

However, as the temperature of the Universe fell towards the threshold for the production of particles $P$ and $\mathrm{P}^{\prime}$, they were formed in ever decreasing numbers so that their annihilation by pairs became prepon- derant. At the threshold temperature, they disappeared from the scene, with the very important exception of protons and neutrons which remained to constitute the material component of the Universe. This was the first key moment when the temperature crossed the proton threshold of $10^{13} \mathrm{~K}$.

Today the residual energy of the Universe, in the form of $3 \mathrm{~K}$ radiation, is unable to synthesize any massive particle, and matter, probably of one type only, can no longer be transformed into energy. Einstein's relation $E=m c^{2}$ is thus now of symbolic significance only, on the cosmic scale.

\section{Condensation of Matter}

It is now believed that while protons and antiprotons were initially formed in equal numbers, and were simultaneously annihilating each other, they could also decay in the physical conditions obtaining immediately after the big bang. Because of charge-parity violation, the rate of decay of the antiprotons was higher than that of the protons. Consequently, at the threshold temperature of $10^{13} \mathrm{~K}$, there was an excess of protons of about 1 in $10^{9}$, but this excess formed the essential matter of the Universe, once pair annihilation was completed.

The proton (or antiproton), it is postulated, is not completely stable but can

Fig. 1 - Friedmann models of the Universe

(A) hyperbolic (infinite Universe)

(B) parabolic (infinite Universe)

(C) elliptic (finite Universe)

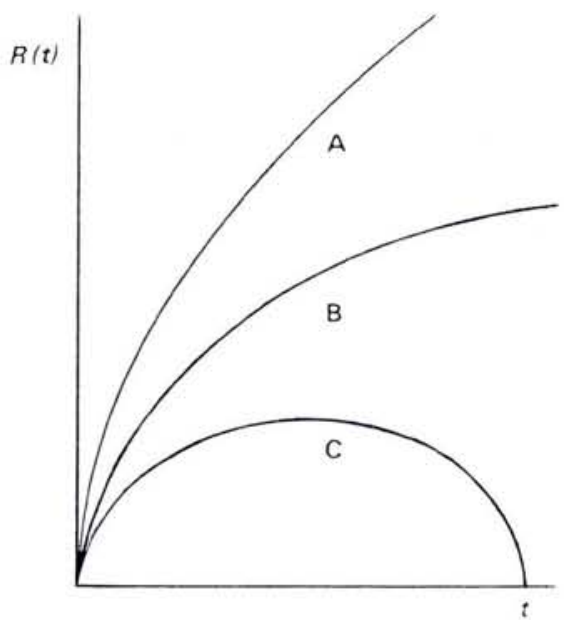


decay through an intermediate particle $X$ of very high mass $\left(10^{15} \mathrm{~m}\right)$. In the present physical conditions of the Universe, this particle has a very low probability of existence in the statistical energy fluctuations of space-time, governed as they are by the indeterminacy principle. Sakharov and Weinberg have calculated that the life-time of the proton is now between $10^{31}$ and $10^{32}$ years which, in every day terms, is equivalent to perfect stability. However, in the primordial Universe, when the temperature exceeded the threshold temperature for this hypothetical particle $X$, its rate of formation was significant and the decay of protons and antiprotons correspondingly rapid. The marginally different rate explains why only one type of matter is to be found in the Universe.

So far, in order to describe the process of primordial nuclear synthesis, only the proton has been considered, but what has been said of the proton applies equally to the neutron, its partner in the atomic nucleus. Its mass is very close to that of the proton, differing by an equivalent energy of only $1.3 \mathrm{MeV}$ in a total of approximately $940 \mathrm{MeV}$. Its threshold temperature is thus also of the order of $10^{13} \mathrm{~K}$.

Well below this temperature, at say $10^{11} \mathrm{~K}$ (the threshold temperature of the mesons is about $10^{12} \mathrm{~K}$ ), the physical state of the Universe could be described rather easily; it was then $0.02 \mathrm{~s}$ old, very dense $\left(4 \times 10^{12} \mathrm{~kg} / \mathrm{m}^{3}\right)$, homogeneous, containing protons, neutrons, electrons, photons and neutrinos, the last two interacting with the free electrons. Only in the region of $10^{10} \mathrm{~K}$ did the cross section for neutrino-electron interactions become negligible, at which point they too became decoupled entities.

Not until the temperature had dropped to around $3000 \mathrm{~K}$ however, did the photons also become decoupled, following which, matter passed from the ionised state to the neutral state, and the Universe changed from being opaque to being transparent. It was the interaction between photons and free electrons during the ionised state that gave the thermal nature to the radiation component of the Universe. The interaction ceased with the disappearance of the free electrons as matter became neutral and the Universe transparent, and, from that time up to the present day, the thermal nature has been preserved, as is well known, by the expansion of space which has also given it its characteristic temperature of $3 \mathrm{~K}$.

\section{Behaviour of Neutrons}

At the condensation temperature of matter (i.e. $10^{13} \mathrm{~K}$ ), the protons and neutrons existed in equal numbers and this equality was maintained by the reversible weak interactions:

$$
\begin{aligned}
& \mathrm{n}+\mathrm{v}=\mathrm{p}^{+}+\mathrm{e}^{-} \\
& \mathrm{p}^{+}+\bar{v}=\mathrm{n}+\mathrm{e}^{+}
\end{aligned}
$$

The disequilibrium came about at around

\begin{tabular}{|c|c|c|c|}
\hline $\begin{array}{l}\text { Tempe- } \\
\text { rature }\end{array}$ & $\begin{array}{l}\text { Redshift } \\
z\end{array}$ & $\begin{array}{l}\text { Age of the } \\
\text { Universe }\end{array}$ & Events \\
\hline $10^{13} \mathrm{~K}$ & $10^{13}$ & $2 \times 10^{-6} \mathrm{~s}$ & Matter condensation (protons + neutrons) \\
\hline $10^{10} \mathrm{~K}$ & $10^{10}$ & $1 \mathrm{~s}$ & Decoupling of the neutrinos \\
\hline $6 \times 10^{9} \mathrm{~K}$ & $6 \times 10^{9}$ & $10 \mathrm{~s}$ & Electron-positron annihilations \\
\hline $10^{9} \cdot 10^{8} \mathrm{~K}$ & $10^{9}-10^{8}$ & $3-30 \mathrm{~min}$. & Primordial nucleosynthesis of $\mathrm{H}$ and $\mathrm{He}$ \\
\hline $3000 \mathrm{~K}$ & 1000 & $10^{6} \mathrm{a}$ & Decoupling of the photons; transparency of the Universe \\
\hline $3 \mathrm{~K}$ & 0 & $2 \times 10^{10} \mathrm{a}$ & Present state of the Universe \\
\hline
\end{tabular}

Table 1 - Evolution of the Universe

$10^{10} \mathrm{~K}$ with the decoupling of the neutrinos, leaving only the reactions:

$$
\begin{aligned}
& \mathrm{p}^{+}+\mathrm{e}^{-}-\mathrm{n}+\mathrm{v} \\
& \mathrm{n}+\mathrm{e}^{+}-\mathrm{p}^{+}+\bar{v}
\end{aligned}
$$

The threshold temperature for their production being only $6 \times 10^{9} \mathrm{~K}$, electrons and positrons were present, at the decoupling of the neutrinos, in about equal numbers, with a weak excess of electrons of around 1 in $10^{9}$. This excess did not contribute significantly to the production of neutrons, but the slightly greater mass of the neutron resulted in the second reaction being favoured in comparison with the first, and, as the cross section for the second interaction is higher, the population of neutrons began to decline in comparison with the protons, the ratio between the two being given by their statistical mass function:

$$
N_{n} / N_{p}=\exp \left[-\left(m_{n}-m_{p}\right) c^{2} / k T\right]
$$

This is an expression that decreases rapidly from nearly unity over a short interval of $T$, such that, when $k T=1 \mathrm{MeV}\left(T=10^{10} \mathrm{~K}\right)$, $N_{\mathrm{n}} / N_{\mathrm{p}}=0.3$.

It is clear, however, that once the creation of electron-positron pairs ceases and annihilation has caused them largely to disappear, the relative decrease in the number of neutrons stops and this takes place at the threshold temperature of $6 x$ $10^{9} \mathrm{~K}$.

The population relation is then:

$$
N_{\mathrm{n}} / N_{\mathrm{p}}=\mathrm{e}^{-0.5}=0.135
$$

This then was the situation that became "frozen" when the temperature dropped below the electron threshold; the age of the Universe was $10 \mathrm{~s}$. Decay of the free neutrons only could then reduce the ratio but as the lifetime of a free neutron is about $1000 \mathrm{~s}$, this was not a significant factor.

\section{Primordial Nucleosynthesis}

In practice, the neutrons would not remain in a free state but would become trapped in atomic nuclei, the determining interactions being:

$$
\begin{array}{r}
p+n-d+\gamma \\
d+d-{ }^{3} \mathrm{He}+n \\
d+d \rightarrow{ }^{3} \mathrm{H}+p \\
{ }^{3} \mathrm{H}+d-{ }^{4} \mathrm{He}+n \\
{ }^{3} \mathrm{He}+\mathrm{d}-{ }^{4} \mathrm{He}+\mathrm{p}
\end{array}
$$

All these interactions are reversible and if we denote the abundance of a nucleon relative to their total number by $A$ :

$$
A_{\mathrm{d}}=N_{\mathrm{d}} / N ; A_{\mathrm{p}}=N_{\mathrm{p}} / N ; A_{\mathrm{n}}=N_{\mathrm{n}} / N
$$

where $N$ is the density of the nucleons, statistical mechanics indicates that:

$$
A_{\mathrm{d}} \sim A_{\mathrm{p}} A_{\mathrm{n}} N\left[\exp \left(E_{\mathrm{d}} / k T\right)\right] /(k T)^{3 / 2}
$$

where $E_{\mathrm{d}}$ is the binding energy of the proton and neutron in deuterium.

Despite, therefore, the reversibility of the first interaction shown above, deuterium could exist in significant quantities when the slope of the exponential took it over the power function $(k T)^{3 / 2}$. This happened as the temperature dropped from about $10^{9}$ to $10^{8} \mathrm{~K}$.

The five interactions listed above, all give rise either directly or eventually to the formation of helium, the process being globalIy equivalent to:

$$
d+d \rightarrow{ }^{4} \mathrm{He}
$$

The abundance of ${ }^{4} \mathrm{He}$ is given by:

$$
A_{\mathrm{He}} \sim A_{\mathrm{d}}^{2} N^{3}\left[\mathrm{I} /(k T)^{3 / 2}\right]^{3} \exp \left(E_{\mathrm{He}} / k T\right)
$$

where $E_{\mathrm{He}}$ is the binding energy of the nucleons of $\mathrm{He}$. At the temperatures under consideration, it proves to be much bigger than for deuterium. The formation of helium is thus strongly favoured by comparison with the other nuclei which exist only as trace elements in the Universe. This result leads to the conclusion that virtually all the primordial free neutrons would have been captured in the formation of helium and the primordial synthesis of nuclei was essentially restricted to building this element. The nuclear synthesis of heavier elements was a subsequent step and of stellar origin.

Such a conclusion seems to be well proved by astronomical observations based on spectral measurements of stellar photospheres which have still today the chemical composition of the primordial clouds out of which the stars were formed. Those products of nuclear reactions which could affect appreciably the chemical composition are, in the main, trapped in the core of stars out of reach of surface convective phenomena, while the anomalous abundances found in some stars can be readily explained on the basis of diffusion mixing. Indeed, the observations of photospheres indicate an abundance of ${ }^{4} \mathrm{He}$ of around $24 \%$ which corresponds closely to the figure that would be deduced from the frozen neutron abundance derived above. This, it will be recalled, was $N_{n} / N_{p}=0.135$ corresponding roughly to $12 \%$ neutrons against $88 \%$ 
protons. With the formation of ${ }^{4} \mathrm{He}$, each neutron is bound to a proton, bringing the helium abundance to $24 \%$ against $76 \%$ for hydrogen. The correspondence is impressive.

All these phenomena developed in the primordial Universe over a very short time scale, as shown in Table 1.

The best expression for dating the successive temperatures $T(t)$, seems to be: $R\left(t_{0}\right) / R(t)$ where $t_{0}$ is the present age of the Universe, since from the relation $R T=c t e$, we obtain:

$$
\mathrm{T}(\mathrm{t}) / \mathrm{T}\left(\mathrm{t}_{\mathrm{o}}\right)=R\left(t_{\mathrm{o}}\right) / R(t)
$$

where $T\left(t_{0}\right)=3 \mathrm{~K}$

The age itself, in seconds or in years, is obtained by the choice of the function $R(t)$, according to a Friedmann model or with the help of the total density of the Universe at this moment - a function of the temperature $T(t)$. Moreover, the well known relation for cosmological redshift $z$ :

$$
1+z=R\left(t_{0}\right) / R(t)
$$

shows finally that, for large values of $R(t) / R(t)$, the value of $T(t)$ is a good approximation for the redshift of a cosmic object which could be hypothetically observed in that phase of the Universe. As an example, it may be considered that matter condensation has a redshift of $10^{13}$, whereas the largest redshifts observed today (in quasars) are three or four only!

Are the fascinating phenomena of the primaeval Universe observable? Perhaps, if to-morrow, astrophysicists develop a neutrino astronomy or a graviton astronomy. But today, the answer is unfortunately negative, as optically, a physical horizon exists at $z=1000$ and $T=3000 \mathrm{~K}$, the photon opacity of the Universe.

\section{BIBLIOGRAPHY}

1. Théories cosmologiques - XXe cours de perfectionnement de l'Association Vaudoise des Chercheurs en Physique; Zermatt, avril 1978. (Observatoire de Genève).

2. Weinberg S., The First Three Minutes. A modern view of the origin of the Universe, (Basic Books, New York) 1977.

3. Weinberg S., "The Decay of the Proton", Scientific American 244 (1981) p. 52.

\title{
Hydrogen Production by Water Electrolysis
}

\author{
S. Stucki, Baden
}

(Research Centre of BBC, Brown Boveri \& Co. Ltd.)

Hydrogen gas is used in large quantities as a raw material in chemical industries. The present need of $\sim 25 \mathrm{Mt} / \mathrm{a}$ is almost entirely extracted from hydrocarbon fuels by reaction with water. The driving force for the reaction in the steam reforming process which liberates four moles of $\mathrm{H}_{2}$ from one mole of $\mathrm{CH}_{4}$ and two moles of $\mathrm{H}_{2} \mathrm{O}$, is part of the energy stored in the natural gas. With the increasing cost of fossil fuels, processes will become competitive which produce hydrogen from water using nuclear or solar energy as the driving force.

The enthalpy change $\Delta H$ for the water splitting reaction

$$
\mathrm{H}_{2} \mathrm{O} \rightarrow \mathrm{H}_{2}+1 / 2 \mathrm{O}_{2}
$$

equals $270 \mathrm{KJ} / \mathrm{mol}$ at room temperature. This high energy input for the production of $1 \mathrm{~mol}$ of hydrogen $(2 \mathrm{~g})$ is one reason why hydrogen has been discussed as a potential synthetic fuel for future energy systems: hydrogen has the highest energy storage density by weight achievable with a chemical fuel. Moreover hydrogen, burnt to form water is an environmentally clean fuel.

\section{Physicochemical Background}

According to chemical thermodynamics, the enthalpy $\triangle H$ is composed of a free enthalpy term $\triangle G$ and an entropy term $T \triangle S$ :

$$
\Delta H_{(T L}=\Delta G_{(T)}+T \Delta S_{(T)}
$$

$\triangle G_{(T)}$ and $T \triangle S$ denominate the relative amounts of work and heat that are thermodynamically necessary for the reaction to proceed at a given temperature $T$. Fig. 1 shows how $\triangle H$ and $\triangle G$ depend upon temperature; the energy scale is in electron volts and corresponds to the electrochemical potential scale. The free enthalpy decreases with temperature, indicating that at temperatures high enough (>3000 K) $\triangle G$ becomes negative and direct thermal water splitting would be possible, provided resistant materials and a high temperature energy source were available.

The most straightforward way to overcome the positive $\triangle G$ at reasonable temperatures is by driving the reaction in an electrochemical cell, consisting of two electrodes immersed in an aqueous electrolyte (Fig. 2). A current flowing in the direction indicated in Fig. 2 produces oxygen at the anode and hydrogen at the cathode. The cell voltage is a function of the current density

$$
U=U^{0}+\eta_{\mathrm{a}}(i)+\eta_{\mathrm{c}}(i)+R i
$$

where $\eta_{a}, \eta_{c}$ are overvoltages due to reactions at the electrodes. Extrapolation to zero current yields the reversible cell voltage $U^{\circ}$ which gives the free enthalpy $\triangle G$ of the reaction. $U^{\circ}$ at room temperature for the water splitting reaction is $1.23 \mathrm{~V}$ (see Fig. 1).

Electrolysis is thermodynamically possible at cell voltages above $1.23 \mathrm{~V}$ at room temperature. The rate of electrolysis depends on the functions $\eta_{3}$ and $\eta$. describing the kinetics of the electrode reaction at the anode and cathode, and on the ohmic resistance $R$ of the cell. The difference between the actual and reversible cell voltages multiplied by the current $i$ gives the amount of electricity which is transformed into heat. As the process itself consumes heat $(T \triangle S)$, some of the waste

Fig. 1 - Dependence on temperature of the thermodynamic functions $\Delta H, \triangle G$, and $T \triangle S$ for the water splitting reaction.

\section{Johannes Kepler University Linz}

In the Faculty of Technical and Natural Sciences (Technisch-Naturwissenschaftliche Fakultăt) of the Johannes Kepler University, Linz, Austria, the position is vacant for a

\section{Full Professor in Biophysics}

The candidate for this post will be expected to devote his research activities to experimental membrane biophysics as his main effort. His teaching should be focused on courses which enable graduate students in physics, chemistry, computer sciences and mathematics to acquire knowledge in biophysics. He should be proficient in German.

Applications including a curriculum and a list of publications should be sent before 30 October 1981, to:

Prof. Dr. Bruno Buchberger

Dean (Dekan) of the Faculty,

Altenbergerstr. 69, A-4040 Linz

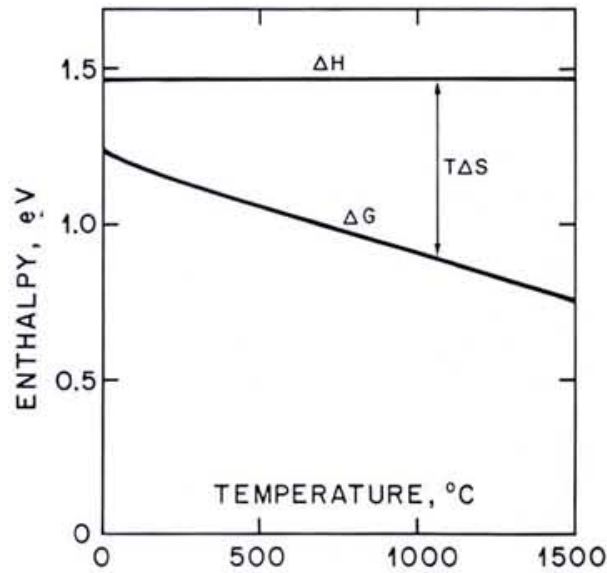

